\title{
Coexisting Kinetically Distinguishable Forms of Dialkylglycine Decarboxylase Engendered by Alkali Metal Ions ${ }^{\dagger}$
}

\author{
Xianzhi Zhou, Sophie Kay, and Michael D. Toney* \\ Department of Biochemistry, Albert Einstein College of Medicine, 1300 Morris Park Avenue, Bronx, New York, 10461
}

Received December 8, 1997; Revised Manuscript Received February 16, 1998

\begin{abstract}
The pyridoxal phosphate (PLP) dependent enzyme dialkylglycine decarboxylase (DGD) specifically binds alkali metal ions near the active site. Large ions $\left(\mathrm{Rb}^{+}, \mathrm{K}^{+}\right)$activate the enzyme while smaller ones $\left(\mathrm{Na}^{+}, \mathrm{Li}^{+}\right)$inhibit it. Crystallographic results have shown that DGD undergoes a metal ion size dependent structural switch [Hohenester, E., Keller, J. W., and Jansonius, J. N. (1994) Biochemistry 33, 13561], but no evidence for multiple conformations in crystalline DGD was obtained. Here, evidence is presented that DGD- $\mathrm{K}^{+}$in solution exists in two conformations differing in catalytic competence. Initial rate traces for DGD- $\mathrm{K}^{+}$exhibit a high degree of curvature due to decreasing activity over time. DGD remains tetrameric under the assay conditions as demonstrated by gel filtration experiments, arguing against the possibility of subunit dissociation as the source of activity loss. Likewise, the mass spectrum of DGD shows a single covalent form. A hysteretic model that assumes two slowly interconverting enzyme forms accounts well for the initial rate data when kinetic parameters from biphasic pre-steady-state kinetics are employed. The fit of the model to the data yields an estimate of $59 \pm 1 \%$ for the fast form. A cooperative model cannot account for the data. Double reciprocal plots for coenzyme binding to DGD exhibit two linear phases. Similarly, two kinetic phases are observed in PLP association kinetics. The substitution of $\mathrm{Na}^{+}$or $\mathrm{Rb}^{+}$for $\mathrm{K}^{+}$alters the steady-state kinetic parameters of DGD. Preincubation of DGD- $\mathrm{K}^{+}$with the competitive inhibitor 1-aminocyclopropane-1-carboxylate (ACC) lowers both $k_{\text {cat }}$ and $K_{\text {AIB }}$ apparently by drawing the enzyme toward the less active, tighter binding form observed in the presteady-state kinetics. These results suggest that the structure of the protein around the alkali metal ion determines the conformational distribution. The transamination reaction with L-alanine was coupled in the pre-steady-state to the LDH-catalyzed oxidation of NADH. This experiment yields an estimate of 68 $\pm 4 \%$ for the fast form, in agreement with the hysteretic fit to the steady-state data. The reaction of DGD with dithiobis(nitrobenzoate) was used to probe the preexisting forms of DGD. Preincubation of DGD with ACC, like the exchange of $\mathrm{Na}^{+}$for $\mathrm{K}^{+}$, shifts the conformational distribution, in agreement with the steady-state kinetics. These experiments clearly demonstrate that DGD is a hysteretic enzyme whose conformational distribution is controlled by the identity of the alkali metal ion bound near the active site, and that cooperativity does not play a role in catalysis or regulation.
\end{abstract}

2,2-Dialkylglycines occur naturally as major constituents of fungal peptide antibiotics $(1,2)$. The catabolic processing of these amino acids in soil bacteria (3) and fungi (4) occurs through the pyridoxal $5^{\prime}$-phosphate (PLP) ${ }^{1}$ dependent 2,2dialkylglycine decarboxylase. DGD catalyzes the oxidative decarboxylation of 2,2-dialkylglycines in the first halfreaction of its ping-pong kinetic mechanism, forming DGD-

\footnotetext{
† Supported by Grant NIGMS 54779 from the National Institutes of Health.

* To whom correspondence should be addressed. Telephone: 718 430-2347. Fax: 718-430-8565. E-mail: toney@aecom.yu.edu.

${ }^{1}$ Abbreviations: DGD, dialkylglycine decarboxylase; PLP, pyridoxal phosphate; PMP, pyridoxamine phosphate; DGD-PLP, PLP form of DGD; DGD-PMP, PMP form of DGD; DGD-K ${ }^{+}$, DGD with potassium bound in metal binding site 1 ; DGD-Na ${ }^{+}$, DGD with sodium bound in metal binding site 1 ; DGD- $\mathrm{Rb}^{+}$, DGD with rubidium bound in metal binding site 1 ; AIB, 2-aminoisobutyric acid; $2^{\circ} \mathrm{ADH}$, NADPHdependent secondary alcohol dehydrogenase from Thermoanaerobium brockii; LDH, lactate dehydrogenase from pig heart; TEA, triethanolamine; Bicine, $N, N$-bis(2-hydroxyethyl)glycine; TMA, tetramethylammonium ion; DTNB, dithiobis(4-nitrobenz-2-oic acid); ACC, 1-aminocyclopropane-1-carboxylate.
}

PMP, carbon dioxide, and a ketone. The second half-reaction is a classical transamination reaction in which the amino group of PMP is transferred to an $\alpha$-keto acid acceptor, preferentially pyruvate, yielding L-alanine and regenerating DGD-PLP.

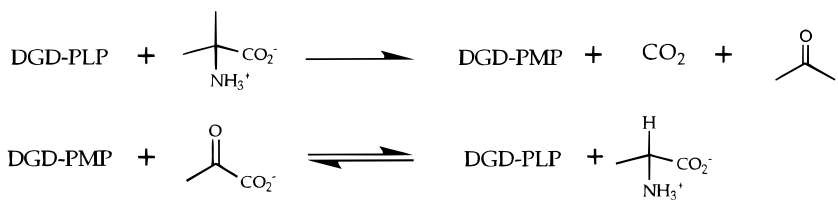

This enzyme is bifunctional in the sense that two classically PLP-dependent reactions, decarboxylation and transamination, are accomplished within a single active site during its normal catalytic cycle.

Structures of DGD in two forms differing in metal ion content (DGD-K ${ }^{+}$and DGD-Na ${ }^{+}$) have been determined by $\mathrm{X}$-ray crystallography (5), and refined and analyzed in detail (6). It is a tetramer, consisting of four identical subunits 
with a mass of $46.5 \mathrm{kDa}$ each based on the gene sequence. The structure revealed two alkali metal ion binding sites: one near the active site binds a variety of alkali metal ions and appears to regulate catalytic activity (site 1), while a second at the $\mathrm{C}$-terminus of an $\alpha$-helix on the surface of the molecule is specific for sodium ions (site 2).

The alkali metal ion binding site near the active site (site 1) has been further studied crystallographically by Hohenester et al. (7). Based on two additional structures (DGD- $\mathrm{Rb}^{+}$ and $\mathrm{DGD}-\mathrm{Li}^{+}$), these authors found a metal ion size dependent two-state switch in which the smaller ions $\mathrm{Li}^{+}$ and $\mathrm{Na}^{+}$cause a concerted rearrangement of two active site residues (Ser80 and Tyr301), as well as smaller global changes that correlate with the inhibitory activity of these metal ions.

There is no crystallographic evidence for the coexistence of multiple conformations in DGD homogeneously liganded by metal at site 1 (e.g., DGD- $\mathrm{K}^{+}$); the different metal ion forms of DGD each appear to be conformationally homogeneous in the crystals. Here, steady-state kinetic and other evidence is presented demonstrating that, in solution, DGD is not conformationally homogeneous; rather, it exists in slowly interconverting conformations that differ in catalytic activity and which may correspond to the crystallographically observed structures of DGD bound to small and large ions (7).

\section{EXPERIMENTAL PROCEDURES}

Materials. Succinic acid, pyruvic acid, sodium chloride, potassium chloride, $\mathrm{PLP}, 2^{\circ} \mathrm{ADH}, \mathrm{LDH}, \mathrm{NADH}$, and NAD$\mathrm{PH}$ were purchased from Sigma. Potassium hydroxide, rubidium hydroxide, TMA hydroxide, TEA, Bicine, DTNB, and AIB were from Aldrich. Ammonium sulfate and L-alanine were from Fisher Scientific. Bio-Rex 70 was from Bio-Rad. PMP was not commercially available. It was prepared by sodium borohydride reduction of PLP dissolved in concentrated ammonium hydroxide. It was purified by ion exchange chromatography on the hydrogen form of BioRex 70 with water as eluant, and then crystallized from ethanol/water.

Enzyme Preparation. The DGD gene was provided by Professor J. W. Keller of the University of Alaska. The gene was PCR-amplified with primers that encode restriction sites allowing direct cloning into pET23a. E. coli BL21(DE3) cells containing pET23a-DGD were grown at $37{ }^{\circ} \mathrm{C}$ in LB medium to an $\mathrm{OD}_{600} \approx 0.9$, induced with $0.5 \mathrm{mM}$ IPTG, and grown $3 \mathrm{~h}$ further at $37^{\circ} \mathrm{C}$. Cells were harvested by centrifugation, and the paste was resuspended in buffer ( 20 $\mathrm{mM}$ TEA-HCl, $\mathrm{pH} 7.8,100 \mathrm{mM} \mathrm{KCl}, 50 \mu \mathrm{M}$ PLP) containing $0.5 \mathrm{mg} / \mathrm{mL}$ lysozyme. Cells were disrupted by sonication, and the homogenate was clarified by centrifugation and brought to $25 \%$ saturation in ammonium sulfate. After centrifugation, the supernatant was brought to $35 \%$ saturation, and the precipitate was redissolved in buffer lacking $\mathrm{KCl}$. Additional buffer was added until the conductivity was equal to that of buffer containing $100 \mathrm{mM} \mathrm{KCl}$. The redissolved precipitate was loaded onto a $2.5 \times 30 \mathrm{~cm}$ column of Q-Sepharose Fast Flow (Pharmacia) which was washed with 1 volume of buffer. Protein was eluted with a linear, $2 \mathrm{~L} \mathrm{KCl}$ gradient $(100-400 \mathrm{mM})$ in buffer at a flow rate of $2 \mathrm{~mL} / \mathrm{min}$. Fractions were assayed for activity and active fractions pooled. DGD elutes at approximately 300 $\mathrm{mM} \mathrm{KCl}$. The pooled enzyme was concentrated to $\sim 10 \mathrm{~mL}$ using an ultrafiltration concentrator (Amicon), and solid $\mathrm{KCl}$ was added to $1 \mathrm{M}$. This sample was loaded on a highperformance Phenyl Sepharose (Pharmacia) column $(1.5 \times$ $15 \mathrm{~cm}$ ). The column was washed with buffer containing 1 $\mathrm{M} \mathrm{KCl}$, and then eluted with a linear $1-0 \mathrm{M} \mathrm{KCl}$ gradient in buffer. The fractions were assayed for activity and analyzed by SDS-PAGE. Those containing pure DGD were pooled, concentrated, flash-frozen, and stored at $-70{ }^{\circ} \mathrm{C}$.

Protein concentration was assayed with the Bio-Rad DC (modified Lowry) assay, using IgG as standard. The TEA background is significant (e.g., buffer absorbance accounts for $34 \%$ of total absorbance at a DGD concentration of 4.7 $\mathrm{mg} / \mathrm{mL}$ ). Both the retained protein solution and the eluate from the ultrafiltration concentrator were diluted severalfold in distilled water before being assayed. Linear plots of $A_{750}$ vs volume of DGD or of eluate were obtained. The slope of these lines along with the slope of the standard line were used to calculate protein concentrations.

Initial Velocity Measurements. These were carried out with a Kontron Uvikon 9420 spectrophotometer. The reaction was assayed by coupling the acetone produced to the $2^{\circ} \mathrm{ADH}$ reaction and monitoring the decrease in NADPH absorbance at $340 \mathrm{~nm}$. Reaction mixtures contained $60 \mathrm{mM}$ Bicine-TMA-hydroxide, $\mathrm{pH}$ 8.2, $100 \mathrm{mM}$ dipotassium succinate, $10 \mathrm{mM}$ sodium chloride, $100 \mu \mathrm{M}$ PLP, $200 \mu \mathrm{M}$ $\mathrm{NADPH}, 1 \mathrm{unit} / \mathrm{mL} 2^{\circ} \mathrm{ADH}$, and varying concentrations of AIB. Pyruvate was obtained as the free acid and titrated with TMA-hydroxide. It was held at $1 \mathrm{mM}$ for DGD-K ${ }^{+}$. Initial rates were taken from absorbance data collected $<2$ min from the time of initiation. Kinetic parameters were estimated by fitting the initial rate data to the MichaelisMenten equation using the program GraFit (Erithacus Software).

$K_{\mathrm{PLP}}$, the concentration of PLP required for half-maximal activation, was measured by incubating DGD for $1 \mathrm{~h}$ with varying concentrations of PLP in $50 \mathrm{mM}$ Bicine, $\mathrm{pH} 8.2$, $100 \mathrm{mM}$ dipotassium succinate, $200 \mu \mathrm{M} \mathrm{NADPH}$, and 1 unit/ $\mathrm{mL} 2^{\circ} \mathrm{ADH}$. Reactions were initiated by addition of substrates in a small volume to final concentrations of 100 $\mathrm{mM}$ AIB and $2 \mathrm{mM}$ pyruvate.

$K_{\text {PMP }}$ was measured with apoDGD prepared by reacting DGD-PLP with an excess of AIB, and then slowly passing it over a $1 \times 30 \mathrm{~cm}$ Sephadex G25 superfine (Pharmacia) column equilibrated with $20 \mathrm{mM}$ TEA $\cdot \mathrm{HCl}, \mathrm{pH}$ 8.0. ApoDGD was incubated $1 \mathrm{~h}$ with various PMP concentrations in the above reaction mixture lacking substrates. Reactions were initiated by addition of substrates in a small volume to final concentrations of $100 \mathrm{mM}$ AIB and $2 \mathrm{mM}$ pyruvate. NADPH absorbance was followed at $380 \mathrm{~nm}\left(\epsilon_{380}\right.$ $=2723 \mathrm{M}^{-1} \mathrm{~cm}^{-1}$ ) to minimize background from PMP.

DGD-Na ${ }^{+}$and DGD- $\mathrm{Rb}^{+}$were prepared by buffer exchange using Centricon-30 concentrators (Amicon). DGDPLP in $50 \mathrm{mM}$ potassium phosphate, $\mathrm{pH} 7.5,75 \mu \mathrm{M}$ PLP, and $300 \mathrm{mM} \mathrm{KCl}$ was exchanged into $50 \mathrm{mM}$ BicineTMA-hydroxide, $\mathrm{pH} 8.2,500 \mathrm{mM}$ disodium or dirubidium succinate, and $100 \mu \mathrm{M}$ PLP. Concentration and 1:10 redilution were repeated $>5$ times over the course of $\sim 5 \mathrm{~h}$. The half-life for dissociation of $\mathrm{K}^{+}$from $\mathrm{DGD}-\mathrm{K}^{+}$was measured to be $\sim 100 \mathrm{~s}$ by observing the decrease in activity when DGD- $\mathrm{K}^{+}$is diluted into a reaction mixture lacking 
metal ions. The binding of $\mathrm{K}^{+}$at high concentrations $(\sim 100$ $\mathrm{mM}$ ) is even faster. Thus, the $\sim 5 \mathrm{~h}$ over which concentration and redilution were carried out is sufficient to ensure complete exchange of metal ions. Additionally, the coenzyme absorbance spectra of the $\mathrm{Na}^{+}$and $\mathrm{Rb}^{+}$forms of DGD were substantially altered from that for DGD- $\mathrm{K}^{+}$. The final concentration of potassium is calculated to be less than 0.01 $\mathrm{mM}$.

$K_{\mathrm{PLP}}$ was measured as above with the substitution of the appropriate metal ion. Saturation curves for AIB were similarly measured in the above reaction mixture in the presence of $2 \mathrm{mM}$ pyruvate and saturating concentrations of PLP (100 $\mu \mathrm{M}$ for DGD-Rb ${ }^{+}, 500 \mu \mathrm{M}$ for DGD-Na ${ }^{+}$).

Gel Filtration To Determine Quaternary Structure. A 1 $\times 25 \mathrm{~cm}$ Sephacryl S-300 (Pharmacia) gel filtration column was equilibrated at room temperature with $50 \mathrm{mM}$ TEA. $\mathrm{HCl}, \mathrm{pH} 7.9,100 \mu \mathrm{M}$ PLP, and $0.1 \mathrm{M}$ of either $\mathrm{KCl}$ or $\mathrm{NaCl}$. A $150 \mu \mathrm{L}$ sample containing $0.4 \mathrm{mg}$ of DGD-PLP and 6 units of malate dehydrogenase in $50 \mathrm{mM}$ TEA $\cdot \mathrm{HCl}, \mathrm{pH} 7.9$, $1 \mathrm{mM}$ PLP, and $0.1 \mathrm{M}$ of either $\mathrm{KCl}$ or $\mathrm{NaCl}$ was applied to the top of the column. The column was developed with the equilibration buffer at a flow rate of $\sim 0.5 \mathrm{~mL} / \mathrm{min}$. Fractions were assayed for absorbance at 278 and $395 \mathrm{~nm}$ (which reports on protein plus PLP, or PLP alone, respectively), and for malate dehydrogenase activity. Malate dehydrogenase was chosen as a standard for the position of the DGD dimer since they are very close in molecular weight. The excess PLP loaded in the sample reports on the position of small molecule elution from the column.

Rate of PLP Binding to ApoDGD. ApoDGD was prepared as above. It was incubated with PLP in a $1 \mathrm{~mL}$ reaction of the following composition: $50 \mathrm{mM} \mathrm{TEA} \cdot \mathrm{HCl}, \mathrm{pH} 7.9,0.1$ $\mathrm{M} \mathrm{KCl}$, either 10, 20, or $40 \mu \mathrm{M}$ PLP, and $\sim 1 \mu \mathrm{M}$ apoDGD. At timed intervals, $100 \mu \mathrm{L}$ of this binding reaction was diluted into $900 \mu \mathrm{L}$ of assay mixture of the following composition: $50 \mathrm{mM} \mathrm{TEA} \cdot \mathrm{HCl}, \mathrm{pH} 7.9,0.1 \mathrm{M} \mathrm{KCl}, 5 \mathrm{mM}$ AIB, $1 \mathrm{mM}$ sodium pyruvate, $1 \mathrm{unit} / \mathrm{mL} 2^{\circ} \mathrm{ADH}, 200 \mu \mathrm{M}$ $\mathrm{NADPH}$, and either 10,20 , or $40 \mu \mathrm{M}$ PLP. The initial rates from these assays were recorded and plotted against time.

Mass Spectral Analysis. The mass spectrum of DGD was obtained on an API-III triple-quadrupole mass spectrometer (PE-SCIEX, Ontario, Canada) using the SCIEX IonSpray interface with nitrogen as the nebulizer gas. An ionspray voltage of $\sim 3600 \mathrm{~V}$ and an orifice voltage of $85 \mathrm{~V}$ were used. The sample was injected to the mass spectrometer at $2 \mu \mathrm{L} / \mathrm{min}$ using a Harvard Apparatus syringe pump after diluting $1: 1$ with $50 \%$ acetonitrile $/ \mathrm{H}_{2} \mathrm{O}+0.1 \%$ TFA.

Pre-Steady-State Coupling of the Reaction of DGD-PLP with L-Alanine to Lactate Dehydrogenase. LDH was obtained as a crystalline suspension in $3.2 \mathrm{M}$ ammonium sulfate. The solid was collected by centrifugation, dissolved in $60 \mathrm{mM}$ Bicine- $\mathrm{KOH}, \mathrm{pH} \mathrm{8.2,} \mathrm{and} \mathrm{dialyzed} \mathrm{against} 2 \mathrm{~L}$ of $60 \mathrm{mM}$ Bicine- $\mathrm{KOH}, \mathrm{pH} 8.2$, for $5 \mathrm{~h}$ at $4{ }^{\circ} \mathrm{C}$. The specific activity was measured after dialysis. Reactions were monitored in a stopped-flow apparatus (Applied Photophysics SX.17MV) at $25{ }^{\circ} \mathrm{C}$. One syringe contained $1.4 \mathrm{mg} / \mathrm{mL}$ DGD-PLP, $60 \mathrm{mM}$ Bicine-KOH, pH 8.2, $250 \mathrm{mM} \mathrm{KCl,} 6$ $\mathrm{mM} \mathrm{NaCl}, 250-2000$ units/mL LDH, and $0.2 \mathrm{mM}$ NADH. The other syringe contained $60 \mathrm{mM}$ Bicine $-\mathrm{KOH}, \mathrm{pH} 8.2$, $250 \mathrm{mM} \mathrm{KCl}, 6 \mathrm{mM} \mathrm{NaCl}, 40 \mathrm{mM}$ L-alanine, 250-2000 units $/ \mathrm{mL} \mathrm{LDH}$, and $0.2 \mathrm{mM} \mathrm{NADH}$. The reaction was monitored at $352 \mathrm{~nm}$ since this is the isosbestic point for the coenzyme absorbance changes observed in the transamination half-reaction.

DGD Reactions with DTNB. For DGD-K ${ }^{+}$, DGD $(1.0 \mathrm{mg} /$ $\mathrm{mL}$ ) in $60 \mathrm{mM}$ Bicine-TMA hydroxide, $\mathrm{pH} 8.2,0.1 \mathrm{M}$ dipotassium succinate was mixed in the stopped-flow apparatus against an equal volume of $2 \mathrm{mM}$ DTNB in the same buffer. DGD- $\mathrm{Na}^{+}$reactions were performed similarly except the sodium salt of succinic acid was used. DGD-K $\mathrm{K}^{+}(1.0$ $\mathrm{mg} / \mathrm{mL}$ ) was preincubated with $40 \mathrm{mM} \mathrm{ACC}$ in the same buffer as for DGD-K $\mathrm{K}^{+}$alone, for $>3 \mathrm{~h}$ at room temperature in the dark. The reactions were monitored at $412 \mathrm{~nm}$ for $1000 \mathrm{~s}$. The reaction of DGD with DTNB over $\sim 1 \mathrm{~h}$ was followed with the Uvikon spectrophotometer. The final DGD concentration was $0.1 \mathrm{mg} / \mathrm{mL}$ ( $1 \mathrm{~mL}$ total volume), with $0.1 \mathrm{M}$ sodium phosphate buffer, $\mathrm{pH} 8.0,1 \mathrm{mM}$ DTNB. An extinction coefficient of $13600 \mathrm{M}^{-1} \mathrm{~cm}^{-1}$ was used for calculating concentrations. The temperature was maintained at $25^{\circ} \mathrm{C}$.

\section{RESULTS}

Steady-State Kinetic Parameters. The initial rate traces for $\mathrm{DGD}-\mathrm{K}^{+}$show large deviations from linearity when monitored over long periods of time (Figure 1A). This nonlinearity is not due to enzyme inactivation since DGD is stable for hours under these conditions, nor is it due to substrate depletion, product inhibition, or the coupling system (Figure 1B). The absorbance data collected over long times are well fit by the equation describing a hysteretic enzyme, eq $1(8)$, as seen by the curves through the data in Figure $1 \mathrm{~A}$.

$$
[\mathrm{P}]=v_{\mathrm{ss}} t-\frac{\left(v_{\mathrm{ss}}-v_{\mathrm{i}}\right)\left(1-\mathrm{e}^{-k t}\right)}{k}
$$

Here, $v_{\mathrm{ss}}$ is the final steady-state velocity, $v_{\mathrm{i}}$ is the initial velocity at the beginning of the assay, and $k$ is the apparent rate constant for isomerization. In this model, two differentially reactive conformations of the enzyme are assumed to coexist, and the equilibrium between the conformers changes over the course of the initial rate measurement. The apparent rate constant, $k$, for the conformational isomerization increases with increasing substrate concentration (Figure 1A).

The kinetic parameters for the reaction of DGD in various forms determined from absorbance data collected in the first $\sim 1$ min of reaction (during which no significant isomerization occurs) are given in Table 1. Double reciprocal plots for these enzymes (Figure 2) show no indication of nonlinearity. Both $K_{\mathrm{AIB}}$ and $k_{\text {cat }}$ values for DGD-K ${ }^{+}$decrease after preincubation with ACC (Table 1; Figure 2). This is not due to the presence of the residual inhibitor $(0.32 \mathrm{mM})$ in the steady-state reactions, which is taken into account in the calculation of the reported parameter values $\left(K_{\mathrm{i}}=1.4 \mathrm{mM}\right.$; S. Sun and M. D. Toney, unpublished results). DGD can bind a variety of alkali metal ions at binding site 1 near the active site $(6,7)$. The data in Table 1 show that both DGD$\mathrm{Na}^{+}$and DGD- $\mathrm{Rb}^{+}$have lower $k_{\text {cat }}$ values than DGD-K ${ }^{+}$, and that the $K_{\mathrm{AIB}}$ and $K_{\mathrm{PLP}}$ values are altered by the metals.

Quaternary Structure of $D G D-K^{+}$and $D G D-N a^{+}$. Gel filtration experiments were performed to examine the quaternary structure of DGD-K ${ }^{+}$and DGD-Na ${ }^{+}$. Figure 3 shows elution profiles indicating that both $\mathrm{DGD}-\mathrm{K}^{+}$and 
Table 1: Steady-State Kinetic Parameters for the Reactions of DGD-PLP with $\mathrm{AIB}^{a}$

\begin{tabular}{lcccc}
\hline & DGD-K & DGD-K $^{+}+\mathrm{ACC}^{b}$ & $\mathrm{DGD}^{+} \mathrm{Rb}^{+}$ & $\mathrm{DGD}^{+\mathrm{Na}^{+}}$ \\
\hline$k_{\text {cat }}\left(\mathrm{s}^{-1}\right)$ & $13.1 \pm 0.2$ & $4.6 \pm 0.1$ & $4.47 \pm 0.03$ & $1.29 \pm 0.02$ \\
$K_{\text {AIB }}(\mathrm{mM})$ & $2.0 \pm 0.1$ & $0.69 \pm 0.04$ & $1.12 \pm 0.03$ & $8.7 \pm 0.6$ \\
$k_{\text {cat }} / K_{\text {AIB }}\left(\mathrm{M}^{-1} \mathrm{~s}^{-1}\right)$ & $6550 \pm 340$ & $6700 \pm 400$ & $3990 \pm 110$ & $150 \pm 10$ \\
$K_{\text {PLP }}(\mu \mathrm{M})$ & $1.5 \pm 0.9$ & - & $5 \pm 2$ & $71 \pm 9$ \\
& $(69 \pm 10)$ & & &
\end{tabular}

${ }^{a}$ Reaction conditions: $\sim 0.1 \mu \mathrm{M}$ DGD, $100 \mu \mathrm{M}$ PLP for DGD-K ${ }^{+}$and DGD-Rb ${ }^{+}, 500 \mu \mathrm{M}$ PLP for DGD-Na ${ }^{+}, 60 \mathrm{mM}$ Bicine-TMA $\cdot$ hydroxide, $\mathrm{pH}$ 8.2, $0.1 \mathrm{M}$ dipotassium succinate, $200 \mu \mathrm{M}$ NADPH, $1 \mathrm{unit} / \mathrm{mL} 2^{\circ} \mathrm{ADH}, 25^{\circ} \mathrm{C}$. The reactions were monitored at $340 \mathrm{~nm}$. The concentration of AIB varied from 0.2 to $20 \mathrm{mM}$, while pyruvate was fixed at either $1 \mathrm{mM}$ (DGD-K ${ }^{+}$) or $2 \mathrm{mM}$ (DGD-Na ${ }^{+}$and DGD-Rb ${ }^{+}$). Initial rate data were fitted to the Michaelis-Menten equation by nonlinear regression (GraFit) to obtain parameter and error estimates. ${ }^{b}$ DGD-K $\mathrm{K}^{+}+$ACC refers to the potassium form of the enzyme that has been preincubated with $40 \mathrm{mM} \mathrm{ACC}\left(K_{\mathrm{i}}=1.4 \mathrm{mM}\right)$ for $>3 \mathrm{~h}$ at $25^{\circ} \mathrm{C}$. The final concentration of $\mathrm{ACC}$ in the reactions was $0.32 \mathrm{mM}$. The kinetic parameters were calculated on the basis of this concentration, the known $K_{\mathrm{i}}$, and the Michaelis-Menten equation with a competitive inhibition term. ${ }^{c}$ Apparent dissociation constant obtained from the dependence of activity on PLP concentration. The binding of PLP and PMP to DGD- $\mathrm{K}^{+}$clearly exhibits nonhyperbolic behavior as shown in Figure 4 . The value in parentheses for DGD-K ${ }^{+}$ corresponds to the weak binding apoenzyme form observed in the nonlinear double-reciprocal plots. $K_{\text {PMP }}$ values of 0.25 and $7 \mathrm{mM}$ were obtained from the data in Figure 4. Double-reciprocal plots for the binding of PLP to the other metal forms of DGD also appeared nonlinear, indicative of two enzymes forms, but regression analysis did not provide reliable estimates for the two forms. The values reported for $\mathrm{DGD}-\mathrm{Rb}^{+}$and $\mathrm{DGD}-\mathrm{Na}^{+}$ are thus for fits to a single enzyme equation.
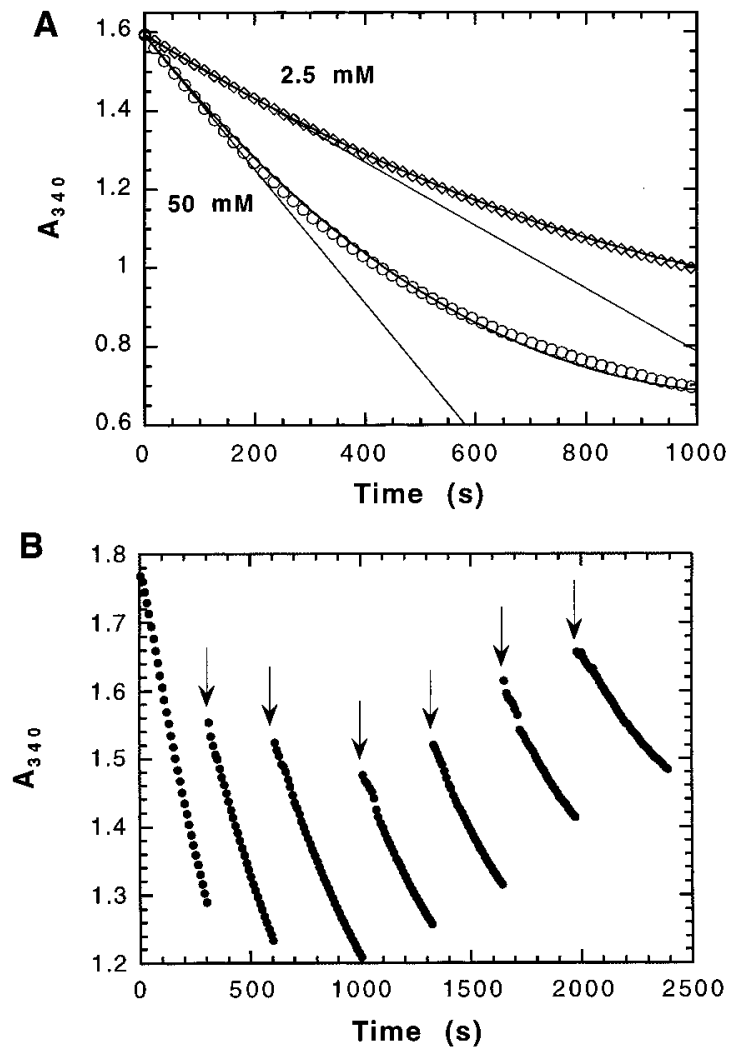

FIGURE 1: (A) Conversion of DGD-K $\mathrm{K}^{+}$from greater to lesser active forms in the course of initial rate measurements made over longer times. Raw traces of $A_{340}$ vs time for reactions containing either $2.5(\diamond)$ or $50 \mathrm{mM}(\bigcirc)$ AIB. The straight lines are drawn through the first few points to emphasize the nonlinearity observed over longer times. The curves through the data were obtained by nonlinear regression with eq 1 , which describes the reaction of a hysteretic enzyme. Reaction conditions: $60 \mathrm{mM}$ Bicine-TMA. hydroxide, $\mathrm{pH} 8.2,100 \mathrm{mM}$ dipotassium succinate, $10 \mathrm{mM}$ sodium chloride, $2 \mathrm{mM}$ sodium pyruvate, $100 \mu \mathrm{M}$ PLP, $200 \mu \mathrm{M}$ NADPH, 1 unit/mL $2^{\circ} \mathrm{ADH}$, varying concentrations of $\mathrm{AIB}, 25^{\circ} \mathrm{C}$. (B) Reaction of DGD-K ${ }^{+}$under conditions similar to those above except that, at the times indicated by the arrows, $5 \mu \mathrm{L}$ aliquots that each gave increases of 0.9 unit of $2^{\circ} \mathrm{ADH}, \sim 50 \mu \mathrm{M} \mathrm{NADPH}$, and 86 $\mu \mathrm{M}$ pyruvate were added to the $1 \mathrm{~mL}$ reaction mixture. The instantaneous initial rates are unchanged by the additions, yet the initial rate decreases over the $\sim 2500 \mathrm{~s}$ during which the reaction was monitored.

DGD-Na ${ }^{+}$are tetrameric under the assay conditions. As expected for tetrameric enzyme, both forms of DGD elute

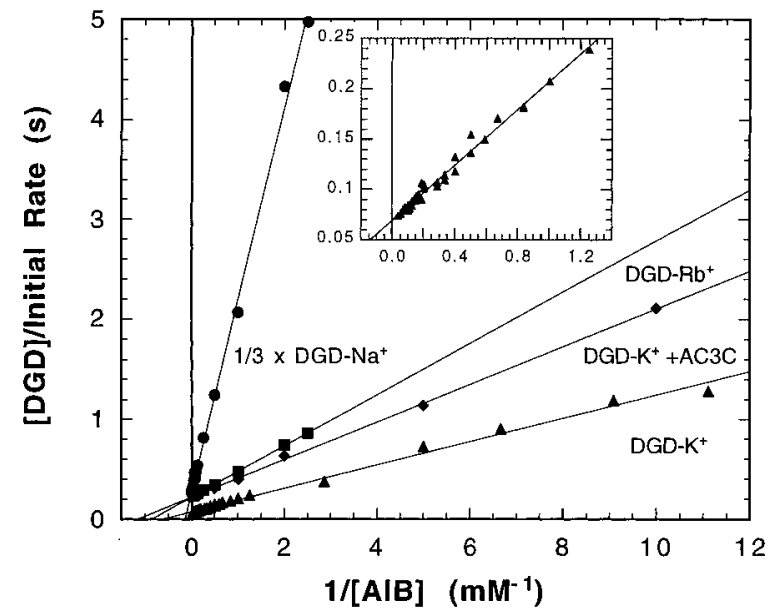

FIGURE 2: Double-reciprocal plot for the reactions of DGD-K ${ }^{+}$ $(\Delta), \mathrm{DGD}^{+} \mathrm{K}^{+}$preincubated with ACC $(\bullet), \mathrm{DGD}^{-\mathrm{Rb}^{+}}(\mathbf{\square})$, and DGD-Na ${ }^{+}(\bullet)$. The reciprocal initial rate values for DGD-Na ${ }^{+}$have been divided by 3 to put them on scale with the others. The inset shows the data for DGD-K ${ }^{+}$at high concentrations of AIB. There is no indication of nonlinearity for any of the enzyme forms within the noise level of the data. Reaction conditions as in Figure 1, except for DGD-Na ${ }^{+}$which contained $500 \mu \mathrm{M}$ PLP.

from the column before malate dehydrogenase, which is nearly equal in size to dimeric DGD.

Dependence of Activity on DGD Concentration. A plot of initial rate (obtained from the first $\sim 1$ min of reaction) vs enzyme concentration exhibits a high degree of linearity ( $r=0.9989$; data not shown) over the concentration range of $1-110 \mathrm{nM}$.

Coenzyme Binding. DGD binds coenzymes relatively weakly, with dissociation constants in the micromolar range. The activation of DGD-K ${ }^{+}$by either PLP or PMP yields nonlinear double-reciprocal plots (Figure 5); two linear regions are observed.

The kinetics of PLP binding were also examined. Figure 6 shows data for the activation of apoDGD by PLP as a function of time. The curves very clearly show two kinetic processes, with the fast essentially complete before the first data point can be taken by the method employed (initial rate measurements as a function of incubation time). The rate constant for the slower phase is proportional to PLP concentration. The variation in the final activity at various PLP concentrations reflects the $K_{\mathrm{PLP}}$ value for DGD-K ${ }^{+}$. 

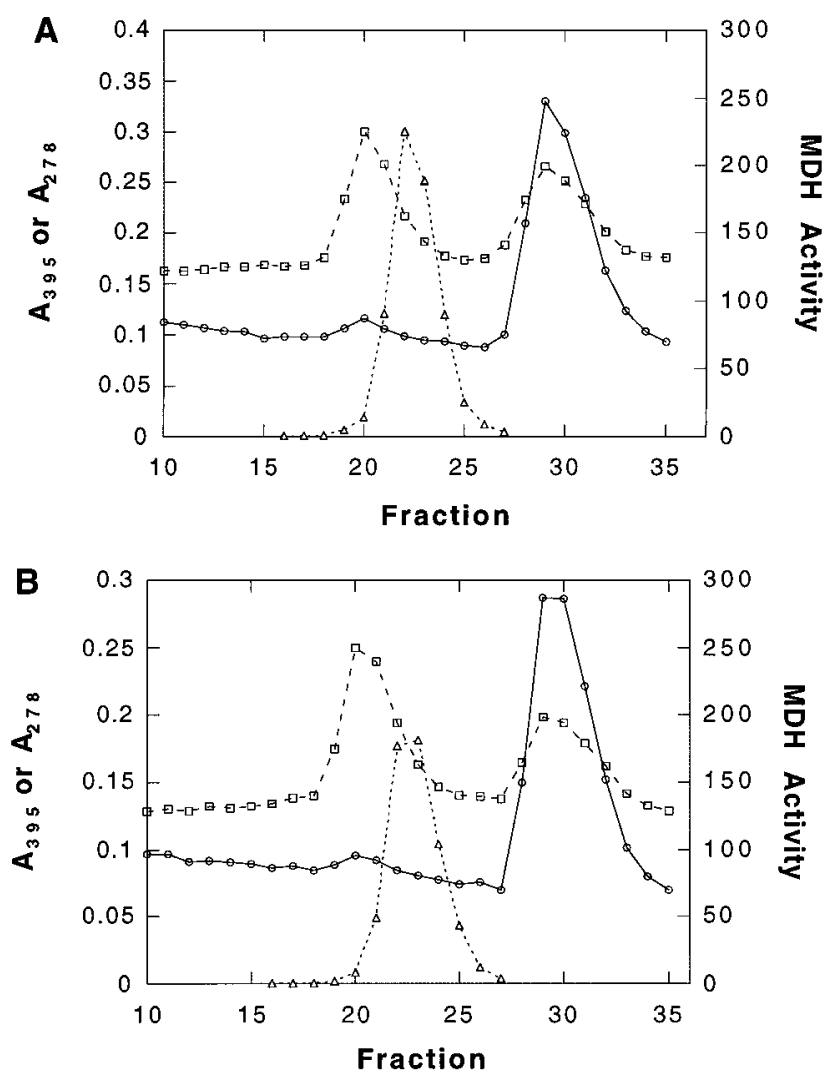

FIGURE 3: Gel filtration elution profiles for mixtures of (A) DGD$\mathrm{K}^{+}$, malate dehydrogenase, and PLP, or (B) DGD-Na ${ }^{+}$, malate dehydrogenase, and PLP. In both cases, the first peak at $278 \mathrm{~nm}$ $(\square)$, corresponding to DGD, elutes before the peak of malate dehydrogenase activity $(\triangle)$. The major peak at $395 \mathrm{~nm}(O)$ corresponds to PLP. Malate dehydrogenase was chosen as a standard since its molecular weight is very close to that of dimeric DGD. Conditions: $50 \mathrm{mM}$ TEA $\cdot \mathrm{HCl}, \mathrm{pH} 7.9,100 \mu \mathrm{M}$ PLP, and $0.1 \mathrm{M}$ of either $\mathrm{KCl}$ or $\mathrm{NaCl}$, room temperature.

Mass Spectrometry. An electrospray ionization mass spectrum was obtained for DGD, which is shown in Figure 7. The mass of 46319 daltons observed for the peak corresponds to the mass of the DGD monomer calculated from the DNA-derived amino acid sequence less the $\mathrm{N}$ terminal methionine. The symmetric nature of the observed peak and the absence of other peaks in this region rule out the possibility that there are multiple covalent forms of DGD present in the preparation.

Coupling of the L-Alanine Transamination Half-Reaction to $L D H$. L-Alanine rapidly undergoes a transamination reaction with the potassium form of DGD-PLP to give DGDPMP and pyruvate. This reaction, as well as the decarboxylation reaction of DGD-PLP with AIB, occurs in two kinetic processes (X. Zhou \& M. D. Toney, in preparation).

It was impractical due to the cost of $2^{\circ} \mathrm{ADH}$ to couple the AIB decarboxylation reaction to NADPH. Instead, the L-alanine transamination reaction was coupled to LDH/ NADH. In this experiment, a large amount of LDH is added so that in the pre-steady-state the reaction of DGD is fully coupled to that of $\mathrm{LDH}$. The decrease in NADH absorbance thus reports on the production of free pyruvate in solution. Figure 8 shows that above $\sim 1000$ units/mL of LDH the system is fully coupled. Clearly, pyruvate is released into solution in two kinetic processes. The two rate constants obtained from the fit of a two-exponential model to the

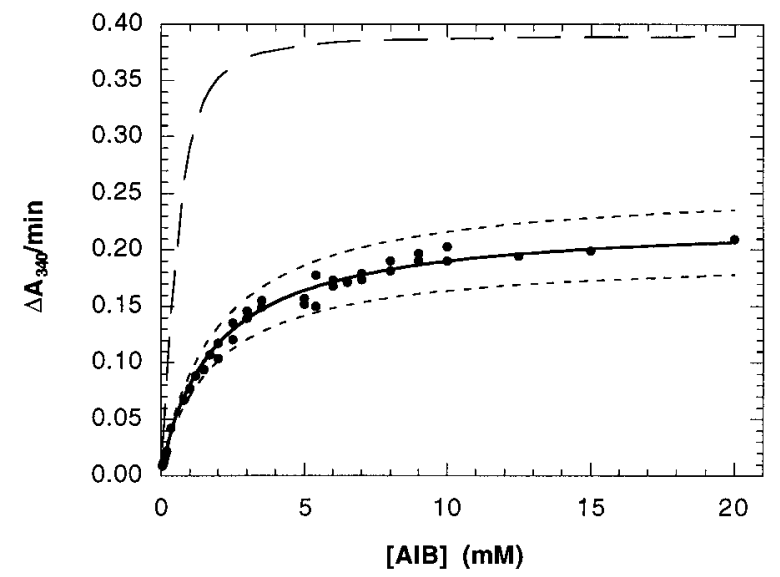

FIGURE 4: Initial velocity saturation curve for the reaction of DGD$\mathrm{K}^{+}$with AIB. The solid curve through the data is the best fit to eq 2 where biphasic pre-steady-state kinetic parameters for the AIB half-reaction were used in place of the steady-state values and the fraction of fast reacting enzyme was the only adjustable parameter. The best-fit estimate of percentage fast form $\left(f_{\text {fast }}\right)$ is $59 \pm 1 \%$. The dashed lines (---) bracketing the data are simulations based on eq 2 and the same kinetic parameters with $f_{\text {fast }}$ set to either $50 \%$ or $68 \%$, and illustrate the sensitivity of the fit to the value of $f_{\text {fast. }}$. The upper dashed line $(--)$ is a simulation based on the cooperative model of Scheme 1 and eq 3 using the same pre-steady-state kinetic parameters and assuming the fast form is cooperatively converted to the slow form. Conditions as in Figure 1.

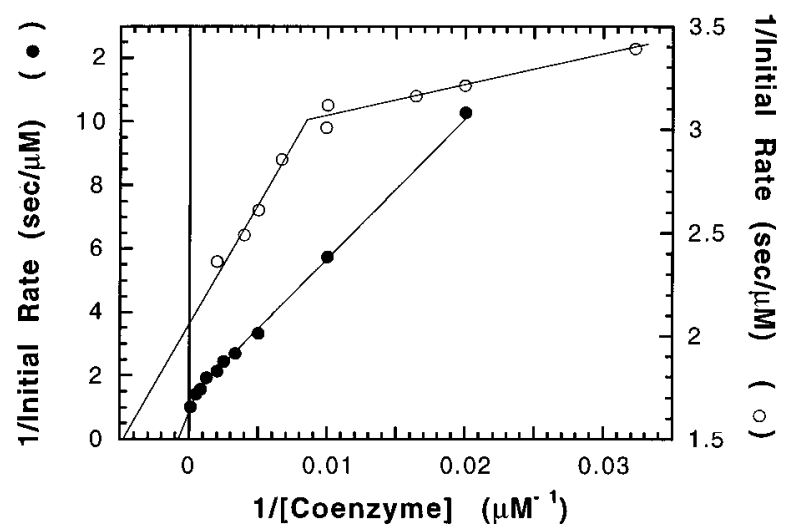

FIGURE 5: Double-reciprocal plots for the activation of DGD-K ${ }^{+}$ by PLP $(\bigcirc)$ or PMP $(\bullet)$. DGD was incubated with various concentrations of coenzymes for $1 \mathrm{~h}$ before the addition of substrates. The equilibrium binding reactions of both coenzymes with DGD-K ${ }^{+}$exhibit two linear regions in the double-reciprocal plots indicative of two apoDGD forms. Reaction conditions: 50 $\mathrm{mM}$ Bicine, $\mathrm{pH} 8.2,100 \mathrm{mM}$ AIB, $2 \mathrm{mM}$ pyruvate, $100 \mathrm{mM}$ dipotassium succinate, $200 \mu \mathrm{M}$ NADPH, 1 unit $/ \mathrm{mL} 2^{\circ} \mathrm{ADH}, 25$ ${ }^{\circ} \mathrm{C}$.

NADH data $\left(18.1 \pm 0.2 \mathrm{~s}^{-1}\right.$ and $\left.0.60 \pm 0.05 \mathrm{~s}^{-1}\right)$ agree well with those obtained from coenzyme absorbance changes $\left(19.6 \pm 0.7 \mathrm{~s}^{-1}\right.$ and $\left.0.52 \pm 0.04 \mathrm{~s}^{-1}\right)$ in the absence of the LDH coupling system under otherwise identical conditions. The average amplitudes for the fast and slow processes obtained from the NADH absorbance change at 1000 units/ $\mathrm{mL} \mathrm{LDH}$ and above are $68 \pm 4 \%$ and $32 \pm 2 \%$, respectively.

$D G D-K^{+}, D G D-K^{+}$Incubated with ACC, and $D G D-N a^{+}$:

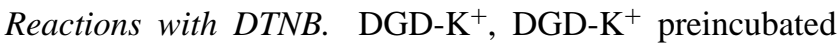
with ACC, and DGD-Na ${ }^{+}$were reacted with DTNB in the stopped-flow apparatus to probe differences in cysteine reactivity due to global conformational differences. Log plots for these reactions are shown in Figure 9. The curve for $\mathrm{DGD}^{+} \mathrm{K}^{+}$preincubated with ACC nearly superimposes 


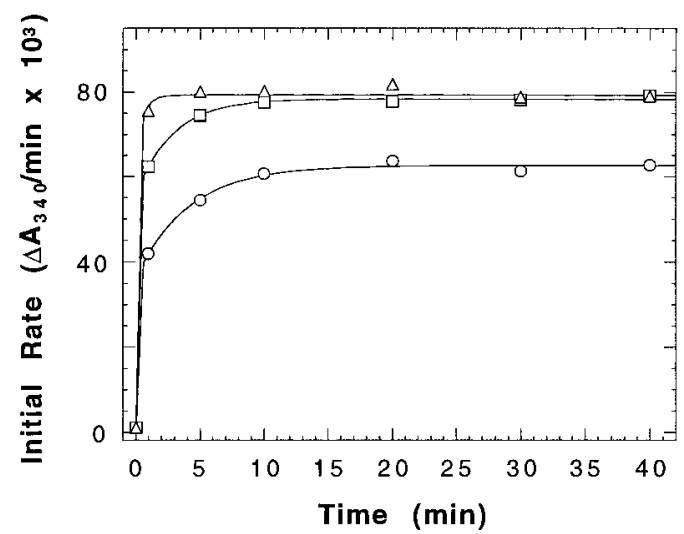

FIGURE 6: Kinetics of the restoration of steady-state activity to apoDGD-K $\mathrm{K}^{+}$by $10(\mathrm{O}), 20(\square)$, and $40(\triangle) \mu \mathrm{M}$ PLP. The reactions are clearly biexponential with the fast phase complete before the first initial rate measurement can be made. The rate constant for the slower phase is dependent on the concentration of PLP. Reaction conditions: $50 \mathrm{mM}$ TEA $\cdot \mathrm{HCl}, \mathrm{pH} 7.9,0.1 \mathrm{M} \mathrm{KCl}$, either 10, 20, or $40 \mu \mathrm{M}$ PLP, $\sim 1 \mu \mathrm{M}$ apoDGD, $25{ }^{\circ} \mathrm{C}$.

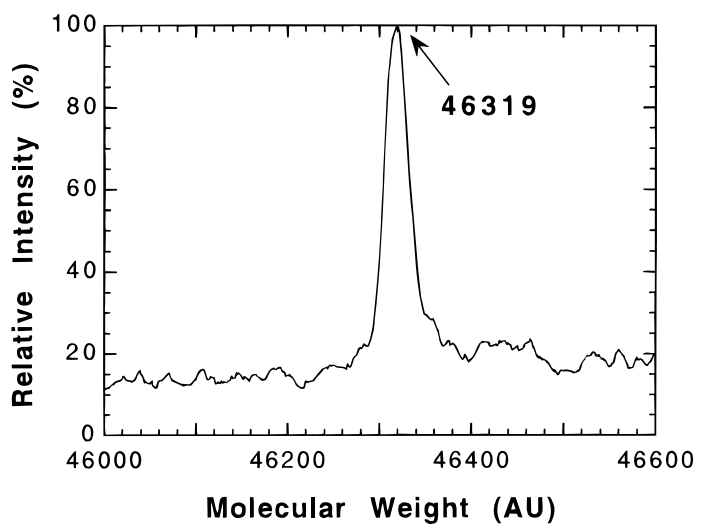

FIGURE 7: Electrospray ionization mass spectrum of DGD. The value of 46319 daltons corresponds to the DGD monomer less the N-terminal methionine. The symmetry of the peak and the absence of other peaks demonstrate that no modified forms exist.

on that for DGD-Na ${ }^{+}$. They are both best described by a two-exponential model. The DGD- $\mathrm{K}^{+}$curve is clearly distinguishable from the others due to the much larger amplitude of its fast kinetic process. The sum of the absorbance changes observed during $\sim 1000 \mathrm{~s}$ corresponds to one cysteine for all enzymes. DGD incubated with $1 \mathrm{mM}$ DTNB in the absence of denaturants reacts three cysteine equivalents over the course of $\sim 2 \mathrm{~h}$ (data not shown).

\section{DISCUSSION}

The concept of enzymatic hysteresis is based on the existence of multiple, slowly interconverting enzyme conformations [or forms, e.g., protonation states (9)]. Neet (8) reviewed this concept and discussed examples from the literature. Several other reviews on the subject are also valuable $(10-12)$.

DGD has been shown crystallographically to exist in one of two conformations when different alkali metal ions are bound near the active site of the enzyme (5). DGD- $\mathrm{K}^{+}$and DGD- $\mathrm{Rb}^{+}$share a similar structure. Likewise, DGD-Na ${ }^{+}$ and DGD- $\mathrm{Li}^{+}$share a structure distinct from that obtained with the larger ions (7). Thus, there is an alkali metal ion size dependent switch in the conformation of DGD in the crystalline state. No evidence for the coexistence of multiple

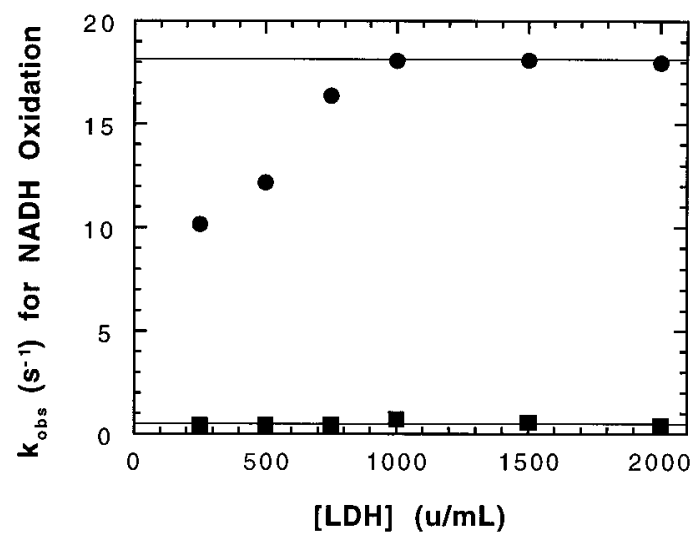

FIGURE 8: Coupling of the transamination reaction between the potassium form of DGD-PLP and L-alanine to the LDH-catalyzed oxidation of NADH. DGD-PLP and L-alanine, both in buffer containing varying amounts of $\mathrm{LDH}$ and $0.2 \mathrm{mM} \mathrm{NADH}$, were mixed in a stopped-flow instrument, and the decrease in absorbance at $352 \mathrm{~nm}$ (the isosbestic point for the coenzyme absorbance changes) was monitored. The reaction in the stopped-flow is fully coupled when the LDH concentration is 1000 units $/ \mathrm{mL}$ or greater. Fast phase rate constants $(\mathbf{O})$; slow phase rate constants $(\mathbf{\square})$. The rate constants obtained from the fit of a two-exponential model to the NADH data at high LDH concentration $\left(18.1 \pm 0.2 \mathrm{~s}^{-1}\right.$ and $0.60 \pm 0.05 \mathrm{~s}^{-1}$ ) agree well with those obtained from coenzyme absorbance changes $\left(19.6 \pm 0.7 \mathrm{~s}^{-1}\right.$ and $\left.0.52 \pm 0.04 \mathrm{~s}^{-1}\right)$ in the absence of the LDH coupling system under otherwise identical conditions. Reaction conditions: $0.72 \mathrm{mg} / \mathrm{mL}$ DGD-PLP, $60 \mathrm{mM}$ Bicine-KOH, $250 \mathrm{mM} \mathrm{KCl}, 6 \mathrm{mM} \mathrm{NaCl}, 20 \mathrm{mM}$ L-alanine, 0.2 mM NADH, varying concentrations of $\mathrm{LDH}, 25^{\circ} \mathrm{C}$.

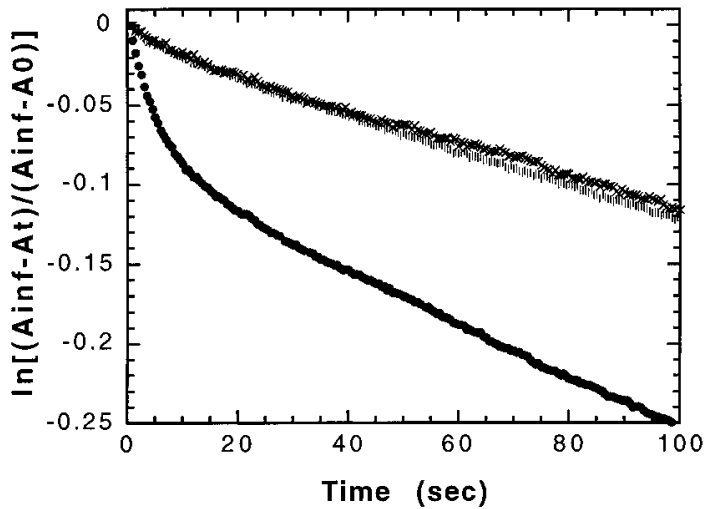

FIGURE 9: Semilogarithmic plot for DTNB reactions with DGD$\mathrm{K}^{+}(\bullet)$, DGD-K $\mathrm{K}^{+}$preincubated with ACC $(\mid)$, and DGD-Na ${ }^{+}(\times)$. Enzyme was mixed against DTNB in a stopped-flow instrument, and the absorbance at $412 \mathrm{~nm}$ was monitored. Reaction conditions: $0.5 \mathrm{mg} / \mathrm{mL}$ DGD, $60 \mathrm{mM}$ Bicine-TMA $\cdot$ hydroxide, $\mathrm{pH} 8.2$, $0.1 \mathrm{M}$ dipotassium succinate, $1 \mathrm{mM}$ DTNB, $25^{\circ} \mathrm{C}$.

conformations in homogeneously metal-liganded crystals was observed. The discussion below demonstrates that homogeneously metal-liganded DGD in solution does exist in multiple conformations, and therefore fulfills the definition of a hysteretic enzyme. The distribution and activity of the conformers in solution are largely determined by the identity of the alkali metal ion bound near the active site.

Nonlinear Progress Curves in Steady-State DGD Reactions Suggest Conversion of a Fast to a Slow Form. The time-dependent loss of DGD activity observed in initial rate traces (Figure 1) could be attributed to several sources including enzyme inactivation, product inhibition, and substrate or coupling system depletion. These potential sources of activity loss were ruled out experimentally by control experiments. For example, in Figure 1B additional coupling 
enzyme, NADPH, and substrate were added at the times indicated by the arrows. The instantaneous initial rate values are not altered by their addition, yet the initial rate decreases in the same time frame as observed in Figure 1A (in which the reaction conditions were unaltered after initiation). Here, a total absorbance change of 1.9 was monitored. DGD-K ${ }^{+}$ activity was measured to be stable for $>4 \mathrm{~h}$ at room temperature under the present conditions.

The loss of activity is likely due to the conversion of a conformation with higher activity to one with lower activity. The observation that the rate constant for the loss of activity is dependent on AIB concentration further suggests that the binding/reaction of AIB is involved in an active site dependent conversion of a fast to a slow enzyme form.

$\mathrm{DGD}-\mathrm{Na}^{+}$and $\mathrm{DGD}-\mathrm{K}^{+}$Are Tetrameric under the Reaction Conditions. The kinetic behavior observed in Figure 1 could arise from the dissociation of the tetrameric form of DGD into a dimeric form. Subunit dissociation has been observed under certain conditions for the PLP-dependent enzyme tryptophanase (13). It is reasonable that a dimeric form of DGD would be active since the tetramer is essentially a dimer of dimers, with the dimer having a structure very similar to that of aspartate aminotransferase (6). Monomers would be inactive since the active sites in the dimer are shared between subunits. The gel filtration data presented in Figure 3 provide strong evidence that both $\mathrm{DGD}-\mathrm{K}^{+}$and DGD-Na ${ }^{+}$do not dissociate into dimers under the conditions of the activity assays. Corroborating this result, the DGD concentration dependence of the initial rate is highly linear $(r=0.9989)$ over a broad concentration range $(1-110 \mathrm{nM})$. The observed kinetic behavior therefore cannot be attributed to the coexistence of dimeric and tetrameric forms of DGD that differ in kinetic properties.

A Two-Enzyme Model Accounts Well for Initial Rates Measured before Significant Isomerization. The half-reaction of the potassium form of DGD-PLP with AIB has been studied by stopped-flow kinetics (X. Zhou and M. D. Toney, manuscript in preparation). This half-reaction fully limits the overall catalytic cycle when pyruvate is the second substrate. Two kinetic processes are observed in the AIB half-reaction with DGD-PLP. Both show hyperbolic dependence on AIB concentration: $k_{\max }=1.2 \pm 0.2 \mathrm{~s}^{-1}, K_{\mathrm{AIB}}$ $=0.24 \pm 0.04 \mathrm{mM}$ for the slow process; and $k_{\max }=23.1 \pm$ $0.4 \mathrm{~s}^{-1}, K_{\mathrm{AIB}}=2.0 \pm 0.1 \mathrm{mM}$ for the fast process.

The steady-state $k_{\text {cat }}$ value observed under the conditions of the half-reaction measurements is $13 \mathrm{~s}^{-1}$ (Table 1). It is thus impossible for the slow process observed in the halfreaction kinetics to correspond to a step in a serial kinetic mechanism for a homogeneous population of enzyme. It must correspond either to the parallel reaction of an independent enzyme form or to a side reaction unrelated to the primary chemistry of the half-reaction itself. The LDH coupling experiments discussed below rule out the latter possibility.

Equation 2 is a kinetic model for two coexisting enzyme forms with different catalytic activities.

$$
v_{\mathrm{i}}=f_{\text {fast }} \frac{V_{\text {fast }}[\mathrm{AIB}]}{K_{\text {fast }}+[\mathrm{AIB}]}+\left(1-f_{\text {fast }}\right) \frac{V_{\text {slow }}[\mathrm{AIB}]}{K_{\text {slow }}+[\mathrm{AIB}]}
$$

Here, $v_{\mathrm{i}}$ is the initial rate, $f_{\text {fast }}$ is the fraction of the fast form, $V_{\text {fast }}$ is the maximal velocity of the fast form, and $K_{\text {fast }}$ is the
Scheme 1: Model for the Cooperative Interconversion of Fast and Slowly Reacting Enzyme ${ }^{a}$

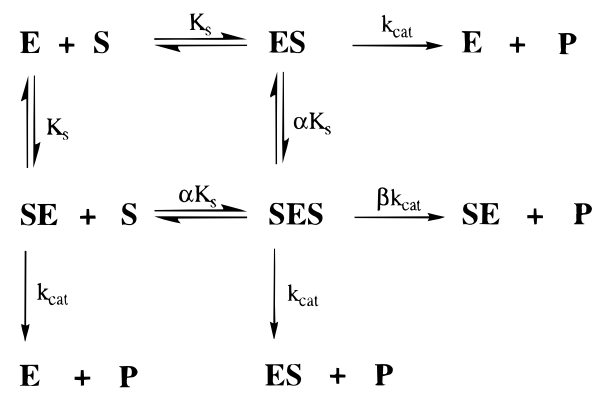

${ }^{a} \alpha$ and $\beta$ are cooperativity parameters that, in the present case, are determined by the ratios of the pre-steady-state parameters observed for the fast and slowly reacting forms of DGD- $\mathrm{K}^{+}$. ES and SE represent substrate bound to different active sites on a multimeric enzyme. Equation 3 is derived from this scheme.

Michaelis constant for the fast form (constants for the slow form are analogously defined). This model should account well for initial rates measured in the first $\sim 1 \mathrm{~min}$ of the DGD reaction, the linear phase in which no significant isomerization between enzyme conformers has occurred.

When the half-reaction kinetic constants for AIB given above are employed with enzyme concentration in eq 2 , it gives an excellent fit (solid curve in Figure 4) to the steadystate data with a value of $f_{\text {fast }}=59 \pm 1 \%$. The small-dashed lines bracketing the experimental data are simulations with eq 2 where $f_{\text {fast }}$ equals $50 \%$ and $68 \%$. These show the high sensitivity of the fit to $f_{\text {fast }}$.

The presence of two kinetic processes in half-reaction kinetics could potentially originate from a cooperative mechanism. The coexistence of two kinetically distinguishable conformers and the presence of cooperativity predict different steady-state kinetics from the same set of presteady-state kinetic parameters. Equation 3

$$
v_{\mathrm{i}}=\frac{2 V_{\max } \frac{[\mathrm{AIB}]}{K_{\mathrm{S}}}+\left(\beta V_{\text {max }}+V_{\text {max }}\right) \frac{[\mathrm{AIB}]^{2}}{\alpha K_{\mathrm{S}}{ }^{2}}}{1+\frac{2[\mathrm{AIB}]}{K_{\mathrm{S}}}+\frac{[\mathrm{AIB}]^{2}}{\alpha K_{\mathrm{S}}{ }^{2}}}
$$

corresponds to the cooperativity model in Scheme 1 (14). Here, $K_{\mathrm{s}}$ is the Michealis constant and $V_{\max }$ is the maximal velocity of the original enzyme. $\alpha$ and $\beta$ are the ratios of $K_{\mathrm{s}}$ and $V_{\max }$ for the original and isomerized enzymes. The simulation based on the pre-steady-state kinetic parameters and eq 3 (Figure 4, large-dashed line) lies far from the experimental data. Here, $\alpha=K_{\text {slow }} / K_{\text {fast }}$ and $\beta=k_{\text {slow }} / k_{\text {fast }}$. The cooperativity model predicts a substantial degree of nonlinearity in the double-reciprocal plots, which, within the noise level of the data presented in Figure 2, would be clearly evident. The two-conformer model, on the other hand, predicts a very small degree of nonlinearity, which would not be detectable in Figure 2. The very poor fit of the cooperativity simulation, the excellent fit of the two-enzyme model, and the absence of nonlinearity in Figure 2 together demonstrate the presence of coexisting, kinetically distinguishable conformational isomers and the absence of cooperative effects.

Coenzyme Binding Suggests Two Forms of ApoDGD. The observation that both PLP and PMP show nonlinear double- 
reciprocal plots for binding to apoDGD (Figure 5) suggests that the existence of multiple conformations is an intrinsic property of the metal-liganded protein. This supports the idea that the structure of the protein around the metal ion at site 1 induces and controls the distribution of conformations (see below). The kinetics of PLP association with DGD occur in two distinct kinetic phases (Figure 6). One is rapid and complete before the first data point can be taken, while the other is slower and dependent on PLP concentration. Two kinetic processes in coenzyme association arise naturally from the existence of two apoDGD conformers.

The Mass Spectrum Demonstrates a Single Covalent Form of $D G D$. The time-dependent steady-state activity loss (Figure 1), the nonlinear double-reciprocal plots for coenzyme binding, and the biphasic association kinetics for PLP with apoDGD might also be explained by the presence of two covalent forms of DGD. One can imagine, for example, the presence of a proteolyzed, less stable form. This possibility was tested by measuring the mass spectrum of the enzyme. Figure 7 shows a single, symmetric peak in the spectrum at 46319 daltons, corresponding to the monomer in which the $\mathrm{N}$-terminal methionine has been removed. Thus, the observed kinetic behavior originates from a single covalent form of the enzyme.

ACC Binding Converts the Fast to the Slow Form. The kinetic parameters for the fast and slow processes observed in the DGD- $\mathrm{K}^{+}$decarboxylation half-reaction with AIB (see above) indicate that the slowly reacting form of the enzyme binds AIB more tightly than the fast form (here, the $K_{\text {app }}$ value is indicative of binding affinity). Thus, one can predict that preincubating DGD- $\mathrm{K}^{+}$with a competitive inhibitor that mimics well the interactions of AIB with the active site would draw the equilibrium toward the tighter binding, slowly reacting enzyme form. Onuffer and Kirsch (15) have demonstrated such an effect with an aspartate aminotransferase mutant in which the aspartate side chain that interacts with the PLP pyridinium nitrogen is replaced with alanine. (This mutant also exhibits a slow interconversion between differentially reactive conformations.) The data in Table 1 and Figure 2 bear out this prediction. $\mathrm{DGD}^{+} \mathrm{K}^{+}$after preincubation with $\mathrm{ACC}$ has a 2.8 -fold reduced $k_{\mathrm{cat}}$ value and a 2.9-fold reduced $K_{\mathrm{M}}$ value. An additional prediction is that the initial rate traces for $\mathrm{DGD}-\mathrm{K}^{+}$preincubated with ACC should be more linear than those for DGD-K ${ }^{+}$not preincubated. This is indeed the case, as it is for DGD-Na ${ }^{+}$ as well (data not shown). These results strongly support the coexistence of multiple slowly interconverting forms and are inconsistent with a cooperativity mechanism.

Distribution and Activity of Conformers Depend on the Identity of Bound Metal Ions. As mentioned above, a dependence of DGD conformation on alkali metal ion size has been observed crystallographically (5). If DGD in solution exists in multiple kinetically distinguishable conformations, then it is likely that different alkali metal ions would alter their distribution. Table 1 and Figure 2 present data for the steady-state reactions of DGD-Na ${ }^{+}$and DGD$\mathrm{Rb}^{+}$that confirm this. These forms both have lower $k_{\text {cat }}$ values than DGD- $\mathrm{K}^{+}$. One might assume that the slowly reacting form of DGD- $\mathrm{K}^{+}$would have the conformation observed crystallographically for DGD- $\mathrm{Na}^{+}$, given the twostate metal ion size dependent structural switch observed crystallographically and the lower $k_{\text {cat }}$ of DGD-Na ${ }^{+}$. The
$K_{\mathrm{AIB}}=8.7 \mathrm{mM}$ for the steady-state reaction of DGD-Na ${ }^{+}$ suggests that this is not the case since the slow form of DGD$\mathrm{K}^{+}$observed in the decarboxylation half-reaction has $K_{\mathrm{AIB}}$ $=0.24 \mathrm{mM}$ (see above). Rather, the lower $k_{\text {cat }}$ and $K_{\mathrm{AIB}}$ for DGD- $\mathrm{Rb}^{+}$compared to DGD-K ${ }^{+}$is consistent with the rubidium form exhibiting the same two conformations observed with DGD- $\mathrm{K}^{+}$, with this metal ion altering the conformational equilibrium toward the lower $k_{\text {cat }}$, lower $K_{\mathrm{AIB}}$ form.

Coupling to LDH Defines the Conformational Equilibrium Constant. The coexistence of two DGD-PLP conformers predicts that two kinetically distinguishable parallel reactions occur when enzyme is mixed with amino acid substrates. The reaction of the potassium form of DGD-PLP with L-alanine, like the reaction with AIB, exhibits two phases in half-reaction kinetics (X. Zhou \& M. D. Toney, manuscript in preparation). The L-alanine half-reaction, which yields pyruvate, was fully coupled in the pre-steady-state to the LDH-catalyzed oxidation of NADH to determine independently the relative amplitudes of the two kinetic processes.

The data in Figure 8 show that at 1000 units $/ \mathrm{mL}$ and above the reaction of the potassium form of DGD-PLP with L-alanine is fully coupled to the LDH reaction. The average amplitudes obtained from this experiment (68\% fast, $32 \%$ slow) provide an independent estimate of the amount of each form. The agreement on $f_{\text {fast }}$ between the fit of the steadystate data to eq 1 (Figure 4) and the LDH-coupled L-alanine reaction $(59 \pm 1 \%$ vs $68 \pm 4 \%)$ convincingly validates the two-conformer model and defines the equilibrium constant to be $\sim 1.8$ in favor of the fast form for DGD-K ${ }^{+}$.

Multiple Conformations Are Evident in DTNB Reactions. The existence of multiple conformers of DGD-PLP was further probed by reactions with DTNB. Two cysteines (Cys258, Cys350) are highly reactive as shown by their preferential modification in the formation of heavy atom derivatives (6). In the absence of denaturants, three cysteines react with DTNB over extended times.

The DTNB reactions of both DGD-K ${ }^{+}$preincubated with ACC and DGD-Na ${ }^{+}$are quantitatively very similar (Figure 9). The reaction of DGD-K $\mathrm{K}^{+}$differs qualitatively from the others in the larger amplitude of the first kinetic process. The sum of the amplitudes of the two kinetic processes equals approximately one cysteine for all three forms, suggesting the differential reactivity of the same cysteine in each conformer is being probed. Preincubation with ACC alters the behavior of DGD-K $\mathrm{K}^{+}$in the DTNB reaction as it alters the steady-state catalytic parameters. Presumably this occurs by the same mechanism: alteration of the conformational equilibrium toward the tight binding form that is catalytically less competent and reacts slower with DTNB. ${ }^{2}$ These results additionally suggest that DGD- $\mathrm{K}^{+}$preincubated with ACC and DGD- $\mathrm{Na}^{+}$largely exist in a single form that resembles the slow form of DGD-K ${ }^{+}$in its overall conformation as probed by cysteine reactivity.

Conclusions. The sum of the data and arguments presented above clearly rule out the presence of cooperativity

\footnotetext{
${ }^{2}$ The observation that both the form of DGD-K ${ }^{+}$that reacts fast with DTNB and the catalytically fast form decrease in percentage with ACC preincubation is serendipitous since the reactions of cysteines far from the active site are determined solely by the local structures around these residues.
} 
between DGD-K $\mathrm{K}^{+}$subunits, and establish the coexistence of slowly interconverting, kinetically distinct conformers. This type of behavior fits the classic definition of a hysteretic enzyme as defined by Frieden (16). The distribution and reactivities of these conformers depend intimately on the identity of the alkali metal ion bound near the active site.

\section{ACKNOWLEDGMENT}

The technical help of Edward Nieves with the acquisition and interpretation of the mass spectral data is gratefully acknowledged. The PMP used here was prepared and generously supplied by Dr. Shaoxian Sun of this laboratory.

\section{REFERENCES}

1. Bruckner, H., Nicholson, G., Jung, G., and Konig, W. A. (1980) Chromatographia 13, 209.

2. Bruckner, H., and Pryzbylski, M. (1984) J. Chromatogr. 296, 263.

3. Bailey, G. B., and Dempsey, W. B. (1967) Biochemistry 6, 1526.
4. Hiura, T., Honma, M., and Shimomura, T. (1978) Agric. Biol. Chem. 42, 1795.

5. Toney, M. D., Hohenester, E., Cowan, S. W., and Jansonius, J. N. (1993) Science 261, 756.

6. Toney, M. D., Hohenester, E., Keller, J. W., and Jansonius, J. (1995) J. Mol. Biol. 245, 151.

7. Hohenester, E., Keller, J. W., and Jansonius, J. N. (1994) Biochemistry 33, 13561.

8. Neet, K. E., and Ainslie, G. R., Jr. (1980) Methods Enzymol. 64, 193.

9. Rose, I. A., Warms, J. V. B., and Yuan, R. G. (1993) Biochemistry 32, 8504.

10. Ricard, J., and Cornish-Bowden, A. (1987) Eur. J. Biochem. 166, 255.

11. Neet, K. E. (1995) Methods Enzymol. 249, 193.

12. Frieden, C. (1979) Annu. Rev. Biochem. 48, 471.

13. Morino, Y., and Snell, E. E. (1967) J. Biol. Chem. 242, 5591.

14. Segel, I. H. (1975) Enzyme Kinetics, p 383, Wiley \& Sons, New York.

15. Onuffer, J. J., and Kirsch, J. F. (1994) Protein Eng. 7, 413.

16. Frieden, C. (1970) J. Biol. Chem 245, 5788.

BI973010U 